\title{
A Gerbil Model of Sloping Sensorineural Hearing Loss
}

\author{
Thomas A. Suberman, Adam P. Campbell, Oliver Franz Adunka, Craig A. Buchman, Joseph \\ P. Roche, and Douglas C. Fitzpatrick \\ Department of Otolaryngology/Head and Neck Surgery, University of North Carolina at Chapel \\ Hill, Chapel Hill, North Carolina, U.S.A.
}

\begin{abstract}
Objective-The goal of the overall project is to develop knowledge about cochlear physiology during cochlear implantation and develop procedures for assessing its status during hearing preservation surgery. As a step toward this goal, for this study, we established an animal model of sloping high frequency sensorineural hearing loss that mimics the hearing condition of candidates for combined electric-acoustic stimulation.

Methods-Mongolian gerbils were exposed to band-pass noise using various cutoff frequencies, intensities, exposure times, and survival times. Hearing loss was assessed in far-field recording using preexposure and postexposure auditory brainstem responses (ABRs), and in acute, near-field recordings of the cochlear microphonic and compound action potential from an electrode on the round window. Anatomic loss of hair cells was assessed from dissections.
\end{abstract}

\begin{abstract}
Results-Postexposure ABRs and near-field recordings from the round window revealed sensorineural hearing loss that varied with the overall noise exposure. Loss of hair cells ranged from relatively sparse to large areas of complete absence depending on the noise exposure. Cases with high intensity ( $120 \mathrm{~dB}$ SPL) and long exposure times $(3 \mathrm{~h}$ ) showed sloping patterns of hearing loss with profound high-frequency loss and mild-to-moderate low-frequency loss. These cases showed complete loss of hair cells in the basal cochlea and preserved hair cells in the apical cochlea. The frequencies comprising the slope in the ABRs and the location of the transition zone between preserved and lost hair cells varied according to the cutoff frequency used.
\end{abstract}

Conclusion-We were able to reliably induce sensorineural hearing loss and loss of hair cells in the gerbil that is comparable to candidates for hearing preservation surgery. This model can be used to evaluate the effects of electrode introduction in a system with a hearing condition similar to that in cases of hearing preservation operations.

\section{Keywords}

Cochlear implant; Electric-acoustic stimulation; Hearing preservation; Hybrid stimulation

\begin{abstract}
Preservation of residual hearing can supplement cochlear implant function by improving speech recognition, especially in the setting of background noise (1-4). Therefore, various surgical techniques have been developed in an effort to preserve hearing remnants during and after cochlear implantation (5-7). Unfortunately, despite these efforts, residual hearing often is compromised, with roughly $50 \%$ of subjects losing at least some portion of their residual hearing capabilities during surgery $(4,8)$.
\end{abstract}

\footnotetext{
Copyright (C) 2011 Otology \& Neurotology, Inc.

Address correspondence and reprint requests to Oliver Franz Adunka, M.D., Otology, Neurotology, Skull Base Surgery, Department of Otolaryngology/Head and Neck Surgery, University of North Carolina at Chapel Hill, P.O. Box, 170 Manning Drive, CB 7070, Chapel Hill, NC 27599-7070; adunka@med.unc.edu.
} 
The underlying mechanisms influencing the success or failure of hearing preservation are not fully understood. However, preserving the morphologic integrity of the organ of Corti is fundamental, and correct placement of the stimulating electrode in a nontraumatic fashion is essential $(5,9,10)$. Previous histologic studies mainly performed in cadaver human temporal bones demonstrated various patterns of intracochlear damage as a result of electrode insertions (11-13). Despite these detailed anatomic data, the functional consequences of intrascalar electrode placement and cochlear damage remain unknown, and intrasurgical monitoring of electrode position and cochlear health is currently unavailable. Therefore, our long-term goal is to measure and map the physiologic correlates of cochlear implant electrode placement.

To this end, we have previously performed a series of experiments testing the effects of electrode interaction with cochlear structures on intracochlear potentials (14-16). In these experiments, we recorded the cochlear microphonic (CM) and the compound action potential (CAP) to acoustic stimuli as an electrode was inserted through the scala tympani. Upon contact with the basilar membrane or osseous spiral lamina, we observed a reduction in the CM and CAP across a wide range of frequencies and intensities (15). With this result, in subsequent experiments, we used only a single stimulus frequency and intensity and showed that even this reduced stimulus set provided a sufficiently sensitive measure of electrode contact that was reversible as the electrode was withdrawn (16). These experiments suggest that an efficient paradigm of cochlear monitoring suitable for intraoperative use can be developed. However, all these experiments were performed in normal-hearing gerbils. Our next steps depend on establishing comparable information in the more clinically relevant setting of sensorineural hearing loss typical in patients using electroacoustic stimulation (EAS). Audiograms in these patients are typically "sloping" in that they consist of a region of profound high-frequency hearing loss and some degree of preserved low-frequency hearing, ranging from normal thresholds to those considered moderate or severe hearing loss. Postmortem cochleograms show complete loss of basal hair cells and no loss or less than complete loss of apical hair cells (17).

Consequently, the aims of this report were to establish an animal model that mimics the EAS condition in terms of a sloping, high-frequency sensorineural hearing loss and extent and distribution of preserved hair cells and to test the availability of acoustically evoked potentials in this setting. Implementing these principles into our model will allow us to study the effects of intracochlear electrode placement in a clinically relevant hearing condition.

\section{MATERIALS AND METHODS}

\section{Animals}

The Mongolian gerbil was chosen for the model because its auditory range is similar to humans, especially with regard to low frequencies (18). Additionally, the gerbil's cranial anatomy, including a large and easily accessible bulla, allows for a relatively straightforward surgical approach and electrode placement. Because of these features, the gerbil cochlea and auditory system in general has been extensively studied (19-21). Fourteen ears from 10 Mongolian gerbils (Meriones unguiculatus) were used for this study. All animals were handled and housed according to the standards described by the National Institutes of Health Committee on Care and Use of Laboratory Animals, using protocols approved by the Institutional Animal Care and Use Committee (IACUC) at the study institution.

\section{Noise Exposure}

The animal was placed in a custom chamber constructed with no parallel walls to minimize standing waves. The animal was suspended in a wire cage in the middle of the sound 
chamber. The speaker (Model D3300Ti; Selenium, Nova Santa Rita/RS, Brazil) was located above the animal. Broadband noise was band-pass filtered with a high cutoff frequency of greater than $20 \mathrm{kHz}$ and a low cutoff frequency of $2,2.5$, or $4 \mathrm{kHz}$. The speaker had a $10-\mathrm{dB}$ low-pass cutoff of $25 \mathrm{kHz}$, so energy above this level was attenuated by the speaker characteristics. Intensities were 116 or $120 \mathrm{~dB}$ SPL, for 2 to 4 hours (Table 1), as measured using a 0.25 -inch microphone and measuring ampli- fier (Brüel and Kjaer, Naerum, Denmark), placed at the position of the cage. After noise exposure, survival times of 2 to 4 weeks allowed for the sequelae of cochlear damage to stabilize (22).

\section{Baseline Recordings and Auditory Brainstem Response}

Auditory brainstem responses (ABRs) were taken before noise exposure and after the survival period. The animal was anesthetized with a ketamine-xylazine $(50 \mathrm{mg} / \mathrm{kg}$ ketamine; $5 \mathrm{mg} / \mathrm{kg}$ xylazine, i.p.) solution. The dorsal portion of the head and the neck were shaved, as well as the fur behind both auricles. Electrodes made of stripped copper wire were then inserted subcutaneously with a needle: an electrode placed at midline slightly anterior to the level of the pinna was connected to the positive lead of the amplifier (Grass Instruments, model P15D), an electrode posterolateral to the right pinna was the return, and the common was placed posterolateral to the left pinna. The tail served as ground. The animal was placed on its left side in a double-walled, sound-attenuated booth, with its right ear facing up (toward the speaker). The animal rested on a chemical heating pad at $39^{\circ} \mathrm{C}$.

ABRs were recorded in response to acoustic stimuli consisting of free-field clicks and tone bursts $(0.5,1,2,4,8$, and $16 \mathrm{kHz})$ delivered from an electrically shielded loudspeaker (Beyer DT48) placed $14 \mathrm{~cm}$ from the animal's eardrum. The speaker had a short sound tube that could be clamped to simulate no-sound recordings to test for the absence of electrical artifacts. Clicks were 100- $\mu$ s duration. Tone bursts had rise and fall times of $2 \mathrm{~ms}$ shaped by a Blackman window with no plateau. Before the experiment, calibration was performed using a 0.25-inch Brüel and Kjaer microphone and measuring amplifier placed at the position of the animal's ear. Intensities were 10 to $90 \mathrm{~dB}$ SPL in 10-dB steps. Each stimulus was presented 500 times at $25 \mathrm{~Hz}$. Recording filters were 10 to $50,000 \mathrm{~Hz}$, and the gain was 1,000. Responses were digitized at $200 \mathrm{kHz}$ and averaged using custom software. Response magnitude was determined as the root mean square voltage in the time window from 1 to 6 ms after the stimulus. The 1-ms delay accounts for acoustic and neural delays, and the 5-ms window accommodates the responses before the middle latency response. The ABR is a saturating response, and threshold was taken as the point where a line tangent to the slope of a logistic fit to the data crossed the noise level. The noise level was taken from a no-stimulus condition in the last $5 \mathrm{~ms}$ of each recording epoch. Objective measures of threshold are not always successful, and in a few cases where the objective threshold estimate gave a clearly erroneous result, the threshold was determined visually.

\section{Surgery and Near-Field Recordings}

Near-field potentials were recorded from the round window after noise exposure and the survival period. Operations were performed under deep urethane anesthesia (25\% solution in saline, $1.5 \mathrm{~g} / \mathrm{kg}$, i.p.). Once anesthesia was induced, the animal was placed in the sound booth, and the body core temperature was monitored with a rectal probe and maintained at approximately $37^{\circ} \mathrm{C}$ with reusable heating pads.

The bulla of the animal was opened, providing exposure to the round window. The recording electrode was attached to a micromanipulator, which allowed for precise placement of the electrode against the intact round window membrane. The electrode was a $50-\mu \mathrm{m}$-diameter Teflon-coated, tungsten-iridium wire with approximately $50 \mu \mathrm{m}$ of insulation removed from the tip. Acoustic stimuli were 100 repetition of free-field tone 
bursts $(0.5,1,2,4,8$, and $16 \mathrm{kHz})$ delivered from the same speaker used during ABR recordings. The tone bursts had 2-ms rise and fall times shaped by a Blackman window and a 10-ms plateau. Intensities were from 3 to $93 \mathrm{~dB}$ SPL in 3-dB steps. Responses were digitized and averaged as described for the ABRs.

The magnitude of the CM response was determined as the amplitude portion of the fast Fourier transform at the stimulus frequency, using the epoch from 6 to $11 \mathrm{~ms}$ where there was no contamination from the CAP. The CM did not saturate at these intensities, so threshold was determined as the point where a line fit to the CM magnitudes crossed the noise level. Noise level was determined from a no-stimulus condition in the last $5 \mathrm{~ms}$ the recording epoch. The magnitude and threshold of the CAP response was determined as previously described for the ABR measurements, after digital band-pass filtering of the recorded signal from 0.5 to $1.5 \mathrm{kHz}$.

\section{Morphologic Assessment}

After completion of an experiment, the animal was killed, and the cochleas were fixed and removed en bloc. Fixation was done by applying $4 \%$ paraformaldehyde in $0.1 \mathrm{M}$ phosphate buffer, ph 7.4, to the oval window on the recorded side after gentle displacement of the stapes, followed by rapid removal of both cochleae and immersion in the fixative. The anatomic extent of the damage was assessed in a preparation where the basilar membrane/ organ of Corti complex was dissected from the spiral ligament and osseous spiral lamina, stained with Weigert's iron hematoxylin (23), mounted on slides, and cover slipped. This preparation was then viewed, analyzed, and photographed for detection hair cell and supporting cell loss or preservation (Figs. 1-3). Photoshop CS3 (Adobe, San Jose, CA, U.S.A.) was used to compile montages of images to create a singular image (Figs. 1A, 2A, and $3, \mathrm{~A}$ and $\mathrm{B}$ ). The anatomic regions of the cochlea were correlated to place-frequency mapping data from the literature (24). Inner and outer hair cells were easily visualized and divided into regions of damage including total, partial, and no hair cell loss. The amount of total hair cell loss (all rows) in the most affected $250-\mu \mathrm{m}$ region was determined from hair cell counts. The loss was determined in the most affected region because total hair cell loss depends on the cutoff frequency used.

\section{RESULTS}

Figure 1A shows the dissected basilar membrane/organ of Corti complex of an unexposed control ear with its tonotopic frequency allocation (24). Using this technique, higher magnification can be used to evaluate the presence of hair cells (Fig. 1, B-D). A comparable case after noise exposure is shown in Figure 2. This ear was exposed to noise with a 4-kHz high-pass cutoff frequency, delivered at $120 \mathrm{~dB}$ SPL for 3 hours (Table 1, Case 63L). The cochlea shows a complete loss of hair cells in basal regions, followed by a transition zone with some hair cells present and complete preservation of hair cells in the apical regions. There is a focal lesion of intense damage at approximately the $14-\mathrm{kHz}$ characteristic frequency position. In Figure 2C, some inner hair cells are missing at the left of the figure, with the rest remaining intact. Outer hair cells are missing across all rows. In general, inner hair cells were more resistant to loss than outer hair cells, as previously reported (25-27).

The location of the transition zone varied depending on the cutoff frequency, as shown for 2 cases in Figure 3. One case (Fig. 3A) is the same as that shown in Figure 2, with the different regions of hair cell loss color coded. The other case had the same exposure (120 dB SPL for $3 \mathrm{~h}$ ) except that the bandwidth was extended so that the high-pass cutoff frequency was $2.5 \mathrm{kHz}$ (Fig. 3B; case 70 in Table 1). In this case, the transition zone (yellow) shifted to a more apical location. A third case had a high-pass cutoff frequency $2.0 \mathrm{kHz}$ with an exposure of $116 \mathrm{~dB}$ for 3 hours (not shown, case 73 in Table 1). The region of completely 
preserved hair cells shifted further toward the apex than in either of the 2 previous cases, consistent with the lower cutoff frequency. However, the lower sound level (by $4 \mathrm{~dB}$ ) combined with speaker's smaller output above $25 \mathrm{kHz}$ produced less than complete loss in the most basal cochlea within approximately $1 \mathrm{~mm}$ of the hook region.

The hearing loss in these cases, as estimated from ABRs, showed the EAS pattern, with profound loss to high frequencies and preservation of hearing at low frequencies. With toneburst ABRs, the position of the slope, or transition from loss of hearing to preserved hearing, varied with cutoff frequency (Fig. 4). This result is consistent with the pattern of hair cell preservation in these cases. The click ABRs showed profound hearing loss, similar to the responses of high-frequency tone bursts.

For the CAP, as with the ABRs, the amount of preserved hearing across frequencies and intensities improved with higher cutoff frequencies (Fig. 5A). Similar results were obtained for the $\mathrm{CM}$ (not shown). At frequencies of $500 \mathrm{~Hz}$ and $1 \mathrm{kHz}$, the $\mathrm{CM}$ is within the band-pass at which the signal was filtered to measure the CAP (see Methods), so the two were incompletely separated by the filtering. The CAP magnitude was still measureable, however, as it was larger than the $\mathrm{CM}$ (as in Fig. 5B). The sources of the $\mathrm{CM}$ and CAP potentials are expected to be quite different between normal-hearing and noise-exposed animals. In normal-hearing animals, a large source of the potentials at high intensities would be from basal hair cells and nerve fibers, which are responding to frequencies in the tails of their tuning curves. In the noise-exposed cases, these hair cells are not present, and the response must be derived from distant, apical hair cells. Despite the distance involved, the recordings of the potentials to low frequencies remained robust when recorded at the round window in noise-exposed animals (Fig. 5B). The smaller responses, as the cutoff frequency is lowered, are expected because the cochlear damage is more extensive, and the responses are from fewer and fewer intact hair cells and auditory nerve fibers. This explanation also would apply to the trend for higher thresholds at low frequencies in the ABRs when the cutoff frequency was lowered (Fig. 4).

In addition to the consistent positions of hair cell loss depending on noise exposure, the hair cell loss also was consistent in the 2 ears of the same animal (Table 1). The results at the round window also were highly similar when both ears from the same case were compared (data not shown).

In other cases (Table 1), the noise exposures were for a shorter duration ( 2 instead of $3 \mathrm{~h}$ ) and 116 instead of $120 \mathrm{~dB}$ SPL. In these cases, the hair cell loss was less than total. We report the maximal hair cell loss in any $250-\mu \mathrm{m}$ region measured. The degree of loss in the most affected region correlated with the extent of damage, in that if only partial loss was observed, the extent of damage was relatively limited, whereas if a total loss occurred, it was over a wide extent of the cochlea. If the hair cell loss was not total at any location, the most affected region was well basal of the center frequency region of the cutoff frequency.

Because of the speaker characteristics, in some cases, there was total loss of hair cells over a wide extent of the basal cochlea, whereas some were still present in the extreme base near the hook.

Overall, the drop in magnitude of the CAP and $\mathrm{CM}$ at $1 \mathrm{kHz}$ correlated well with the percentage of hair cell loss (Fig. 6A, $r=-0.85$ for the CM and -0.83 for the CAP, $p<$ 0.005 ). The correlations for the threshold changes (Fig. 6B) were similar for the CM ( $r=$ $0.8, p=0.003)$ but were not as good for the CAP $(r=0.59, p=0.059)$. This difference between threshold and magnitude for the CAP is presumably related to the overall difficulty in determining threshold in these waveforms. Finally, the change in magnitudes and thresholds were themselves correlated (Fig. 6C, $r=-0.94, p<0.0001$ for the CM, $r=$ 
$-0.66, p=0.027$ for the CAP). Given that magnitude to suprathreshold stimuli can be measured more easily than threshold itself, the relationship between them is useful.

\section{DISCUSSION}

The experiments demonstrate the ability to create particular patterns and severities of sensorineural hearing loss in the gerbil. By modifying the parameters of the noise exposure, we could alter the degree and extent of basal hair cell loss, within the limits of variability associated with noise exposure. The anatomic changes were paralleled by shifts in ABRs and near-field measures of cochlear responses. Some parameter combinations induced a high-frequency, sloping hearing loss that mimics hearing impairments seen in candidates for EAS.

The requirements for a model of sloping hearing loss are a complete loss of auditory input from basal locations associated with profound hearing loss at high frequencies, combined with preserved apical cochlear integrity and low-frequency hearing. This pattern was produced by a high level of sound (120 dB SPL), delivered for 3 hours, with a 1-month survival time. With these parameters, the boundary region (transition zone) between damage and preservation was a function of cutoff frequency. With less exposure, that is, even $4 \mathrm{~dB}$ less intensity and a shorter exposure, there was typically less than complete destruction of hair cells and a high degree of variability (cf., Table 1, Cases 54 and 59). When the goal is complete destruction of a region of hearing, parameters in excess of the minimum required can be used, providing some protection against variability. In addition, the combination of anatomic and physiologic analysis in each case means that the results do not assume that a specific predicted result from the noise exposure was achieved. Instead, the damage and hearing loss are assessed in detail, and the future goal is to correlate the effects of electrode insertion on cochlear integrity and residual hearing capability. The results at this stage demonstrate that the pattern desired can be obtained with the parameters specified.

\section{Comparison With Previous Animal Studies}

In general, the results are in accord with previous animal studies. An important finding for future studies with our hearing loss model is that the extent of cochlear damage in the 2 ears was comparable. This means that 1 ear can be used as a control for the extent of noise damage, whereas the effects of further damage because of cochlear implantation can be explored in the other ear. In an extensive study in chinchillas (25), the correlation (linear regression) of hair cell loss on the 2 sides for inner and outer hair cells was 0.93 and 0.97 , respectively. A main conclusion from that study was that this high degree of correlation allowed for damage because of exposure in one ear to serve as a reliable proxy for damage in the other ear.

In gerbils, exposure to 2-octave noise bands for 1 hour at $120 \mathrm{~dB}$ SPL resulted in loss of hair cells over a restricted range and a large notch in the audiogram (27). Although similar to our results, a notched audiogram does not mimic that used for EAS operations. Studies in other species have used less intense noise and produced a sloping hearing loss, but the hearing loss is typically not profound, and/or the hair cell loss is not complete (27). Complete loss of hair cells to produce profound hearing loss is usually achieved with ototoxic drugs or a combination of drugs and noise (e.g., (28)). However, the effect of ototoxic drugs is nonspecific for frequency, so intense noise exposure is a better choice for our goals. As far as we are aware, ours is the first study to produce a sloping hearing loss model with profound hearing loss, complete loss of basal hair cells and preservation of apical hair cells, and low-frequency hearing that mimics the hearing condition of EAS candidates. 


\section{Comparison of Gerbil and Human Sloping Hearing Loss}

Current guidelines for EAS candidacy require essentially no more than moderate hearing loss at low frequencies and severe or profound hearing impairment at higher frequencies $(4,29)$. This type of hearing loss has been correlated with postmortem histology that reveals preserved apical hair cells and complete loss of basal hair cells (17). In the gerbil, intense stimulation (116-120 dB SPL for $3 \mathrm{~h}$ ) was required to produce similar patterns of hearing loss and cochlear damage. Even at $116 \mathrm{~dB}$, if subjected to only 2 hours of noise, then the loss of hair cells was less than the total at the site of maximal damage, and the hearing loss at high frequencies was not profound (Table 1).

The cutoff frequencies used in our 3 best cases (Figs. 1-5) produced survival of hair cells at apical positions comparable to those of humans. The Greenwood equation can be used to determine the place-frequency map of the human cochlea and with modifications of animal cochleae as well $(30,31)$. The map for the gerbil was determined by Muller (24) and applied to our experimental material (Figs. 1-3). In the human, the upper limit of 1,500-Hz preserved hearing for EAS occurs at approximately $45 \%$ from the apex. In the gerbil cochlea, the frequency that corresponds to this position is approximately $4 \mathrm{kHz}$. In our experiments, the position of the slope in the ABRs and of the transition zone in hair cells showed a good correspondence to the high-pass cutoff frequency. Thus, by using cutoff frequencies of $4 \mathrm{kHz}$ or lower, we are able to produce ranges of hearing loss and hair cell survival that reasonably mimic conditions for EAS.

The size and shape of the gerbil cochlea, however, differs from the human. In particular, at a cochlear distance of approximately $3 \mathrm{~mm}$ from the base, there is a severe constriction in the size of the scala tympani (32). This constriction will limit the ability to insert an electrode beyond that point. Consequently, to physiologically identify a boundary between surviving and nonsurviving hair cells (a goal of subsequent experiments), it may be necessary to use higher cutoff frequencies to place the transition zone at the end of the large basal turn. Another difference in the gerbil model is that the survival times of 2 to 4 weeks are short compared with the time of hearing loss before an EAS surgery. In cases where the hearing loss may have extended for years, the basilar membrane/organ of Corti complex is covered by a relatively undifferentiated epithelium (33). It is not clear what effect, if any, this difference may have on future use of the model.

\section{The Gerbil as a Model to Develop Efficient Intracochlear Recording Techniques During Hearing Preservation Cochlear Implantations}

We are using the gerbil to determine parameters of intracochlear recordings that can potentially be obtained and used during a human electrode insertion. We have previously shown that intracochlear measurements made as an electrode is advanced across the scala tympani show a reduction in response and increase in threshold to auditory stimulation as the electrode encounters sensitive cochlear structures (14-16). The reductions in response occur at a wide range of frequencies and intensities. A large extent of the cochlea responds to suprathreshold stimuli, and the responses are large, so little averaging is required. In subsequent experiments, we showed that a sensitive and rapid measure of electrode contact could be obtained with only a single stimulus frequency at suprathreshold intensity (16). These results suggest that an efficient paradigm of cochlear monitoring suitable for intraoperative use can be developed.

Important steps remain before this conclusion is fully warranted. In particular, the previous experiments used normal-hearing animals. In this most sensitive condition, large responses are expected, and generators at the site of electrode impact will contribute to the potential measured. In the case of a hearing preservation surgery, the hearing condition is insensitive 
across much of the frequency range, and the source of acoustically sensitive potentials is remote from the basal site of electrode insertion. Consequently, it is necessary to determine if large potentials to suprathreshold stimuli are still available as a measure of cochlear integrity. Therefore, the present experiments were initiated to develop and use the gerbil model with a hearing condition similar to that of patients using EAS. Using noise exposure, we were able to produce such a hearing condition, as indicated through hair cell survival, ABRs, and potentials recorded at the round window. Importantly, the responses recorded at the round window were large, although the surviving hair cells were in the apical part of the cochlea. Future experiments testing the effects of electrode interaction with cochlear structures, and identifying the boundary of hair cell survival for optimal electrode placement, can therefore be done using this model.

\section{Acknowledgments}

The authors thank Steven Pulver for excellent technical assistance. The authors also thank Dr. Paul Manis for sharing his expertise.

\section{REFERENCES}

1. von Ilberg C, Kiefer J, Tillein J, et al. Electric-acoustic stimulation of the auditory system. New technology for severe hearing loss. ORL J Otorhinolaryngol Relat Spec. 1999; 61:334-340. [PubMed: 10545807]

2. Kang SY, Colesa DJ, Swiderski DL, Su GL, Raphael Y, Pfingst BE. Effects of hearing preservation on psychophysical responses to cochlear implant stimulation. J Assoc Res Otolaryngol. 2010; 11:245-265. [PubMed: 19902297]

3. Gantz BJ, Turner C, Gfeller KE, Lowder MW. Preservation of hearing in cochlear implant surgery: advantages of combined electrical and acoustical speech processing. Laryngoscope. 2005; 115:796802. [PubMed: 15867642]

4. Gstoettner WK, Van De Heyning P, O'Connor AF, et al. Electric acoustic stimulation of the auditory system: results of a multi-centre investigation. Acta Otolaryngol. 2008:1-8.

5. Kiefer J, Gstoettner W, Baumgartner W, et al. Conservation of low-frequency hearing in cochlear implantation. Acta Otolaryngol. 2004; 124:272-280. [PubMed: 15141755]

6. Berrettini S, Forli F, Passetti S. Preservation of residual hearing following cochlear implantation: comparison between three surgical techniques. J Laryngol Otol. 2008; 122:246-252. [PubMed: 17666134]

7. Turner CW, Reiss LA, Gantz BJ. Combined acoustic and electric hearing: preserving residual acoustic hearing. Hear Res. 2008; 242:164-171. [PubMed: 18164883]

8. Tyler RS, Gantz BJ, Rubinstein JT, et al. Three-month results with bilateral cochlear implants. Ear Hear. 2002; 23:80S-89S. [PubMed: 11883771]

9. James C, Albegger K, Battmer R, et al. Preservation of residual hearing with cochlear implantation: how and why. Acta Otolaryngol. 2005; 125:481-491. [PubMed: 16092537]

10. Fraysse B, Macias AR, Sterkers O, et al. Residual hearing conservation and electroacoustic stimulation with the nucleus 24 contour advance cochlear implant. Otol Neurotol. 2006; 27:624633. [PubMed: 16868510]

11. Adunka O, Unkelbach MH, Mack M, Hambek M, Gstoettner W, Kiefer J. Cochlear implantation via the round window membrane minimizes trauma to cochlear structures: a histologically controlled insertion study. Acta Otolaryngol. 2004; 124:807-812. [PubMed: 15370564]

12. Adunka O, Kiefer J, Unkelbach MH, Lehnert T, Gstoettner W. Development and evaluation of an improved cochlear implant electrode design for electric acoustic stimulation. Laryngoscope. 2004; 114:1237-1241. [PubMed: 15235353]

13. Skarzynski H, Fayette RP. A new cochlear implant electrode design for preservation of residual hearing: a temporal bone study. Acta Otolaryngol. 2010; 130:435-442. [PubMed: 19883172]

14. Campbell AP, Suberman TA, Buchman CA, Fitzpatrick DC, Adunka OF. Flexible cochlear microendoscopy in the gerbil. Laryngoscope. 2010; 120:1619-1624. [PubMed: 20564668] 
15. Adunka O, Mlot S, Suberman TA, et al. Intracochlear recordings of electrophysiologic parameters indicating cochlear damage. 2010 submitted.

16. Campbell AP, Suberman TA, Buchman CA, Fitzpatrick DC, Adunka OF. Correlation of early auditory potentials and intracochlear electrode insertion properties-an animal model featuring near real-time monitoring. Otol Neurotol. 2010; 31:1391-1398. [PubMed: 20856155]

17. Schuknecht HF. Auditory and cytocochlear correlates of inner ear disorders. Otolaryngol Head Neck Surg. 1994; 110:530-538. [PubMed: 8208568]

18. Ryan A. Hearing sensitivity of the Mongolian gerbil, Meriones unguiculatus. J Acoust Soc Am. 1976; 59:1222-1226. [PubMed: 956517]

19. Cant NB, Benson CG. Organization of the inferior colliculus of the gerbil (Meriones unguiculatus): projections from the cochlear nucleus. Neuroscience. 2008; 154:206-217. [PubMed: 18359572]

20. Budinger E, Scheich H. Anatomical connections suitable for the direct processing of neuronal information of different modalities via the rodent primary auditory cortex. Hear Res. 2009; 258:16-27. [PubMed: 19446016]

21. Frisina RD, Chamberlain SC, Brachman ML, Smith RL. Anatomy and physiology of the gerbil cochlear nucleus: an improved surgical approach for microelectrode studies. Hear Res. 1982; 6:259-275. [PubMed: 7045062]

22. Yamashita D, Jiang HY, Schacht J, Miller JM. Delayed production of free radicals following noise exposure. Brain Res. 2004; 1019:201-209. [PubMed: 15306254]

23. Nicol JA. The Weigert-Pal method for paraffin embedded material; decalcification of material containing bone. Can J Res. 1949; 27:4-6. [PubMed: 18112017]

24. Muller M. The cochlear place-frequency map of the adult and developing Mongolian gerbil. Hear Res. 1996; 94:148-156. [PubMed: 8789820]

25. Bohne BA, Bozzay DG, Harding GW. Interaural correlations in normal and traumatized cochleas: length and sensory cell loss. J Acoust Soc Am. 1986; 80:1729-1736. [PubMed: 3794079]

26. Bohne BA, Harding GW. Degeneration in the cochlea after noise damage: primary versus secondary events. Am J Otol. 2000; 21:505-509. [PubMed: 10912695]

27. Ryan A, Bone RC. Noise-induced threshold shift and cochlear pathology in the Mongolian gerbil. J Acoust Soc Am. 1978; 63:1145-1151. [PubMed: 649873]

28. Chen GD, Henderson D. Cochlear injuries induced by the combined exposure to noise and styrene. Hear Res. 2009; 254:25-33. [PubMed: 19371775]

29. Gantz BJ, Hansen MR, Turner CW, Oleson JJ, Reiss LA, Parkinson AJ. Hybrid 10 clinical trial: preliminary results. Audiol Neurootol. 2009; 14:32-38. [PubMed: 19390173]

30. Greenwood DD. A cochlear frequency-position function for several species-29 years later. J Acoust Soc Am. 1990; 87:2592-2605. [PubMed: 2373794]

31. Greenwood DD. Comparing octaves, frequency ranges, and cochlear-map curvature across species. Hear Res. 1996; 94:157-162. [PubMed: 8789821]

32. Thorne M, Salt AN, DeMott JE, Henson MM, Henson OW Jr, Gewalt SL. Cochlear fluid space dimensions for six species derived from reconstructions of three-dimensional magnetic resonance images. Laryngoscope. 1999; 109:1661-1668. [PubMed: 10522939]

33. Li PM, Somdas MA, Eddington DK, Nadol JB Jr. Analysis of intracochlear new bone and fibrous tissue formation in human subjects with cochlear implants. Ann Otol Rhinol Laryngol. 2007; 116:731-738. [PubMed: 17987778] 

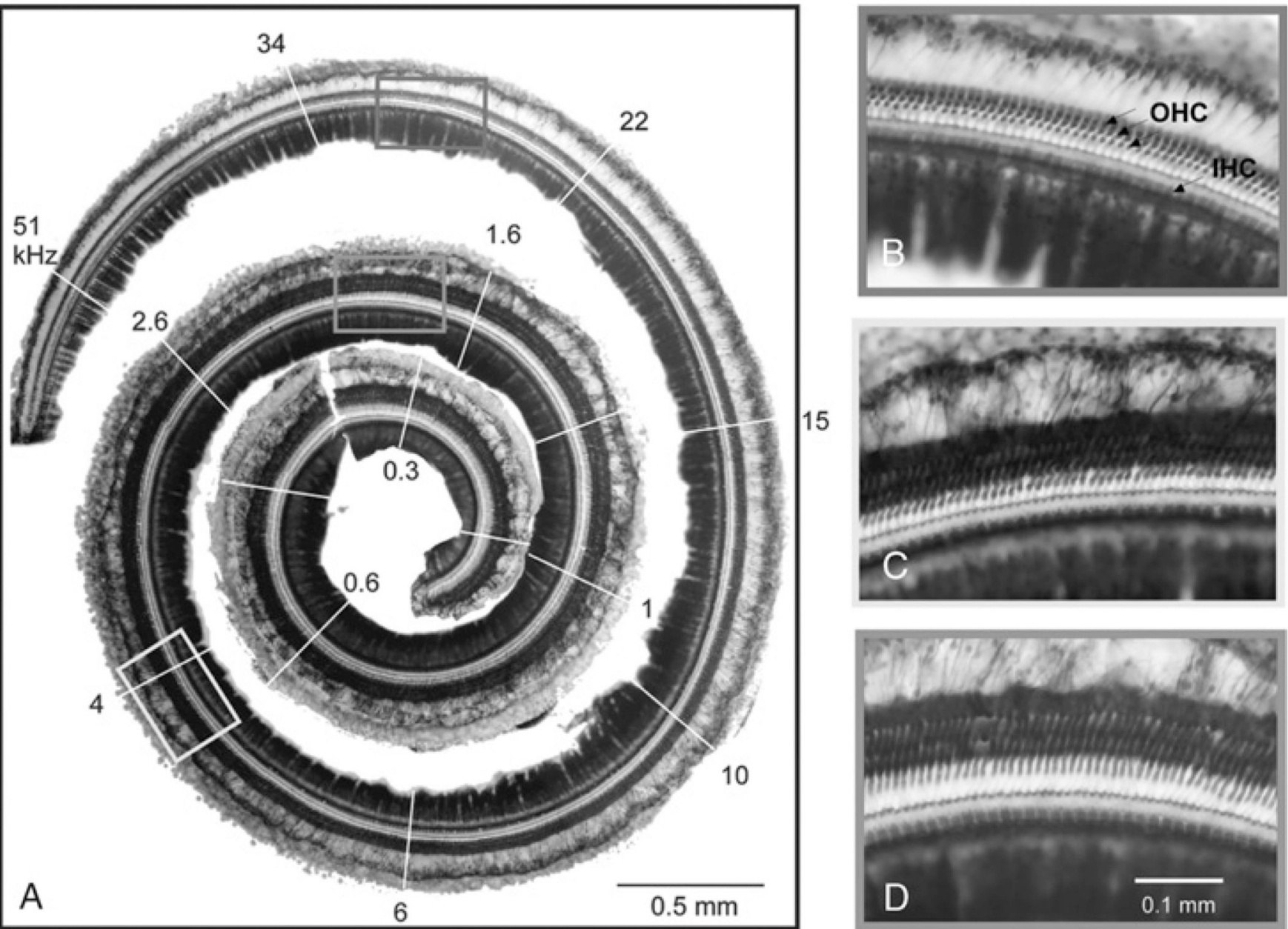

FIG. 1.

Dissected basilar membrane/organ of Corti complex of a normal-hearing gerbil (Gerbil 79). $\boldsymbol{A}$, Photomontage of the entire cochlea showing regions of characteristic frequency (white lines with numbers in $\mathrm{kHz}$, derived from Muller (24)). $\boldsymbol{B}, \boldsymbol{C}$, and $\boldsymbol{D}$, Magnified sections that reveal intact cochlear hair cells and preservation of hair cell organization throughout all regions of the cochlea. Arrows point to outer (OHCs) and inner hair cells (IHCs). 

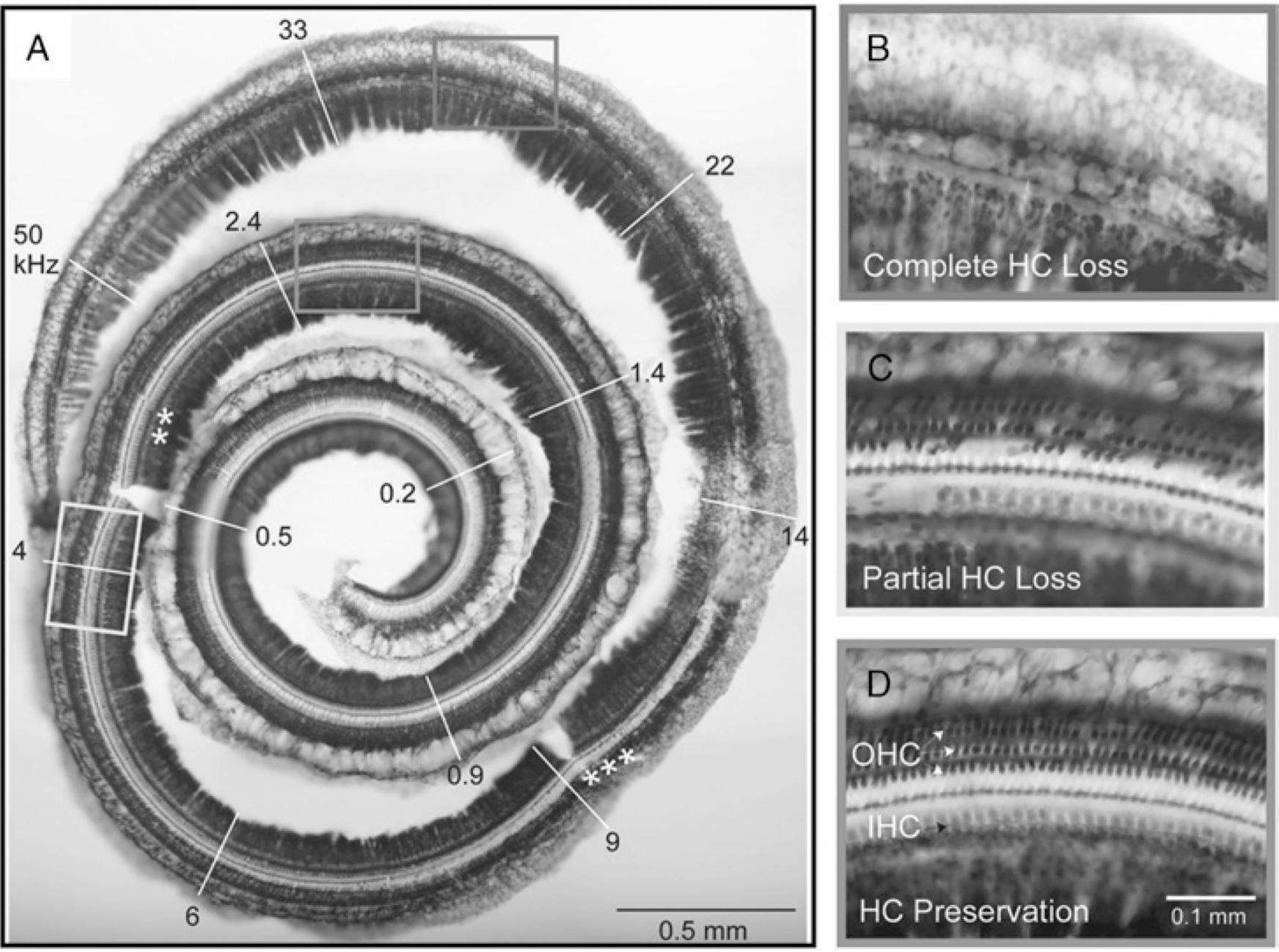

FIG. 2.

Hair cell loss after noise exposure (Gerbil 63). Noise exposure was $120 \mathrm{~dB}$ SPL for 3 hours with a high-pass cutoff of $4 \mathrm{kHz}$. $A$, There is complete loss of hair cells and supporting cells in the organ of Corti extending from the base to the 3 asterisks. Partial hair cell loss extends beyond this to the 2 asterisks. From there, hair cells are preserved to the apex. B, Magnified view of complete hair cell loss. $\boldsymbol{C}$, Magnified view of partial loss of inner and outer hair cells. $\boldsymbol{D}$, Magnified view of apical zone, which contains a nearly normal complement of inner and outer hair cells. 


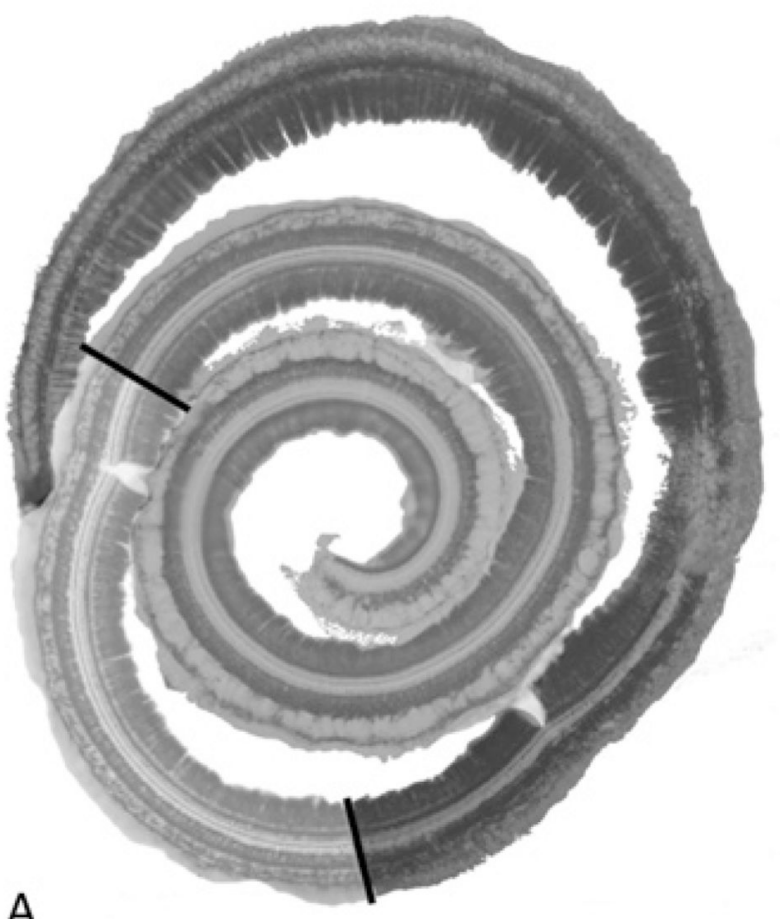

A

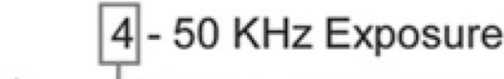

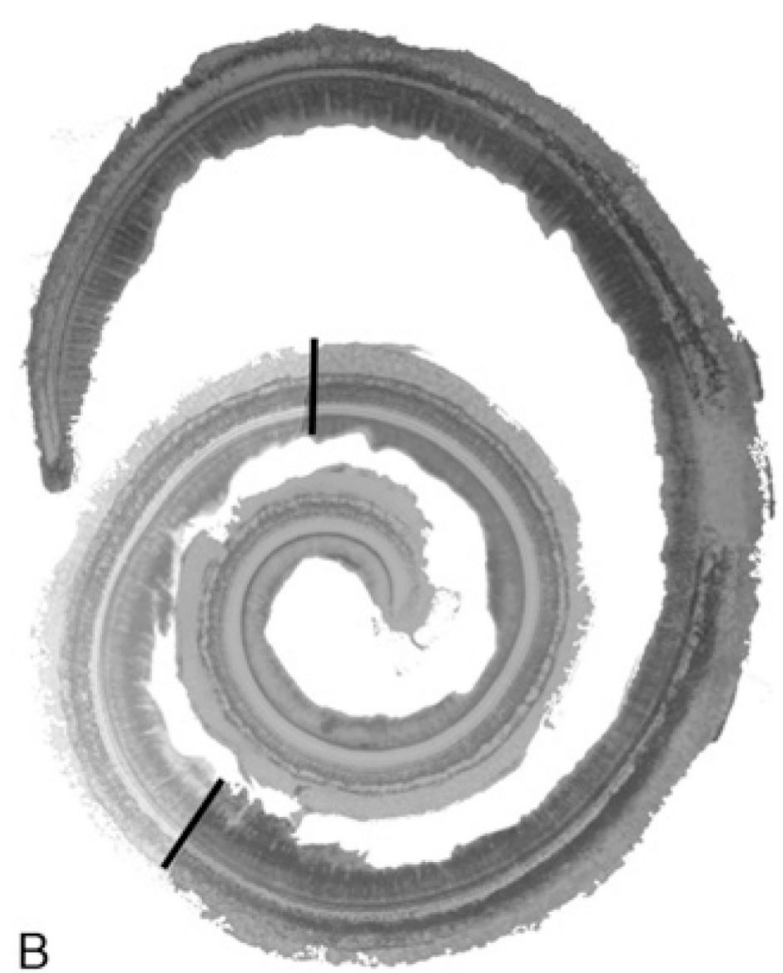

2.5- $50 \mathrm{KHz}$ Exposure

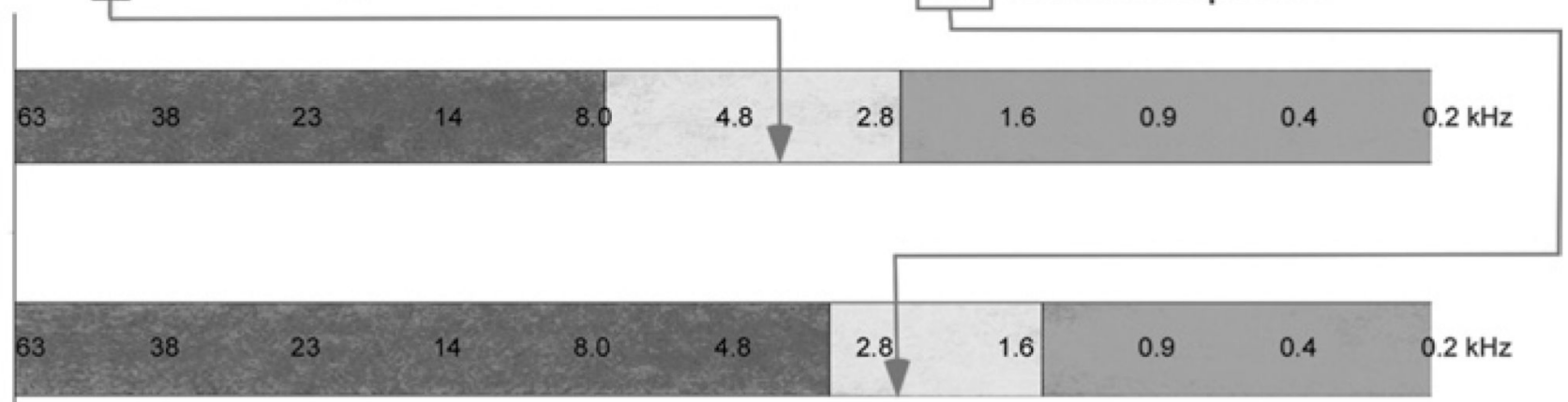

FIG. 3.

Histologic replication of residual hearing in the gerbil model. $\boldsymbol{A}$, Color-coded representation of the extent of the 3 regions defined in the cochlea of Figure 2. $\boldsymbol{B}$, Specimen from a separate case with a high-pass cutoff of $2.5 \mathrm{kHz}$ (Gerbil 70). $\boldsymbol{C}$, The degree of hair cell damage from each case with superimposed tonotopic frequency information. As anticipated, a larger region of complete hair cell loss (red area) is observed with the wider $(2.5 \mathrm{kHz}$ cutoff) noise exposure. The arrows mark the cutoff frequencies for each case, both of which fall within the zone of partial $\mathrm{HC}$ destruction. 


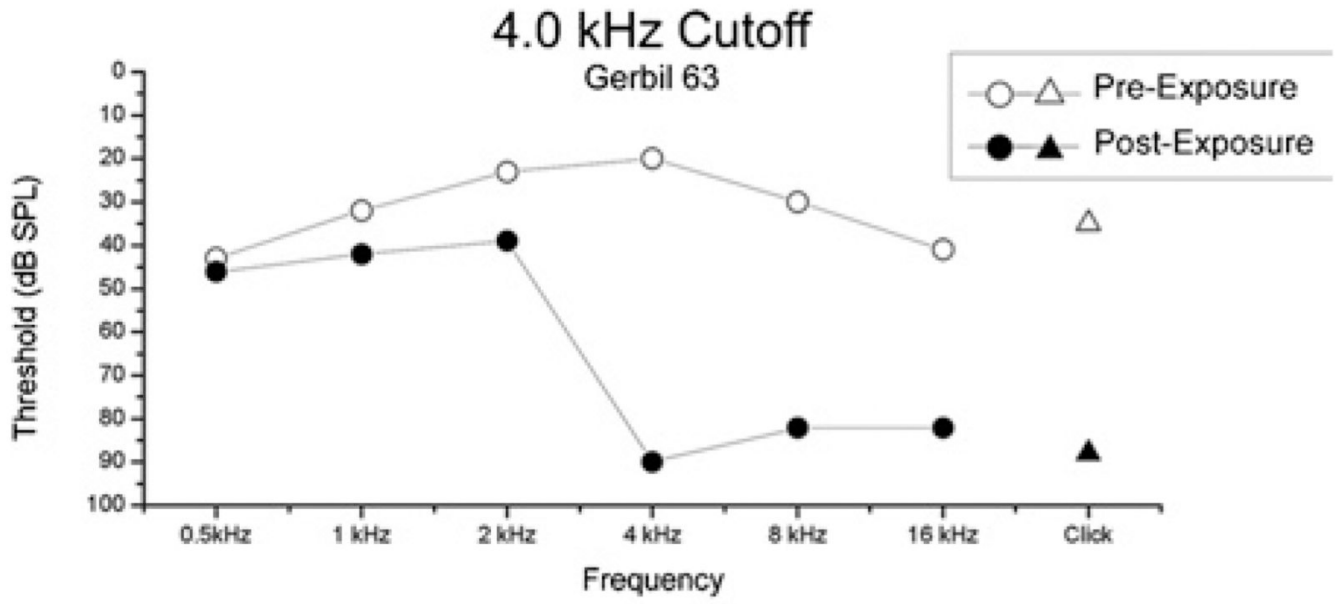

A

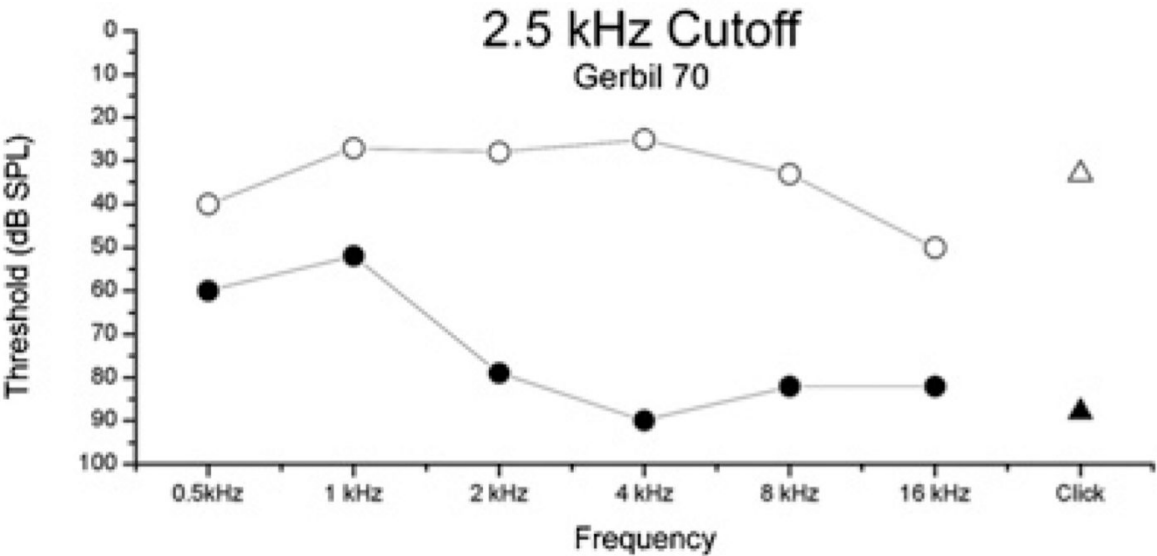

B

Frequency

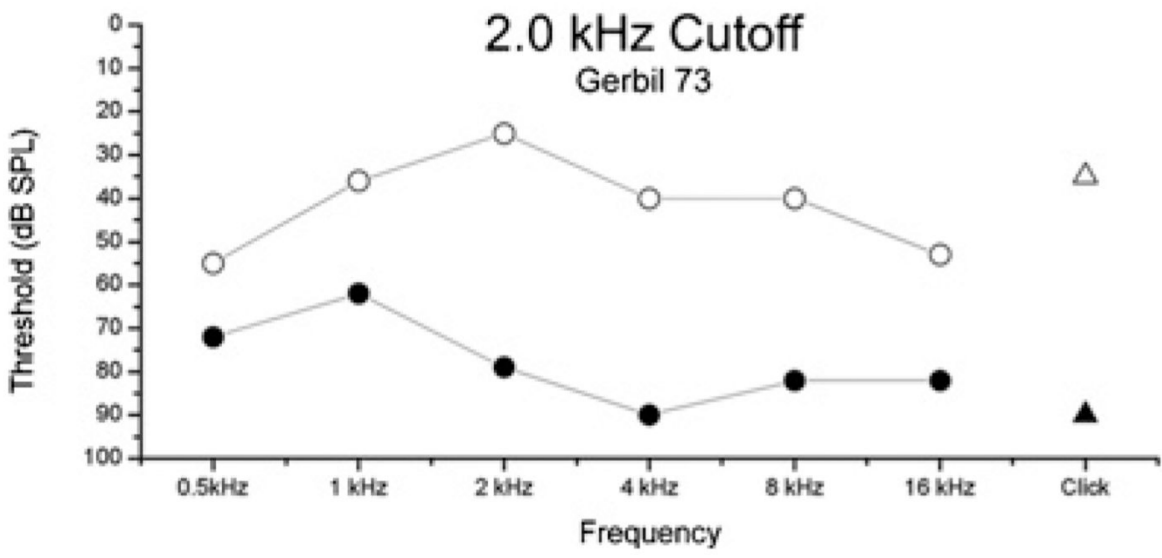

FIG. 4.

ABR measurements showing threshold changes for 3 cases of noise exposure using various high-pass cutoff frequencies. The low-frequency hearing thresholds correlated well with the high-pass cutoff frequency used. The responses to click stimuli (triangles) are severely reduced, indicating they are generated predominately by the basal cochlea. The degree of low-frequency preservation was reduced as the cutoff frequency was lowered, despite the fact that a lower intensity (116 dB SPL versus $120 \mathrm{~dB}$ SPL) was used in the case with a 2.0kHz cutoff frequency. A, Gerbil 63, same case as Figures 2 and 3A. B, Gerbil 70, same case as Figure 3B. $\boldsymbol{C}$, Gerbil 73. 

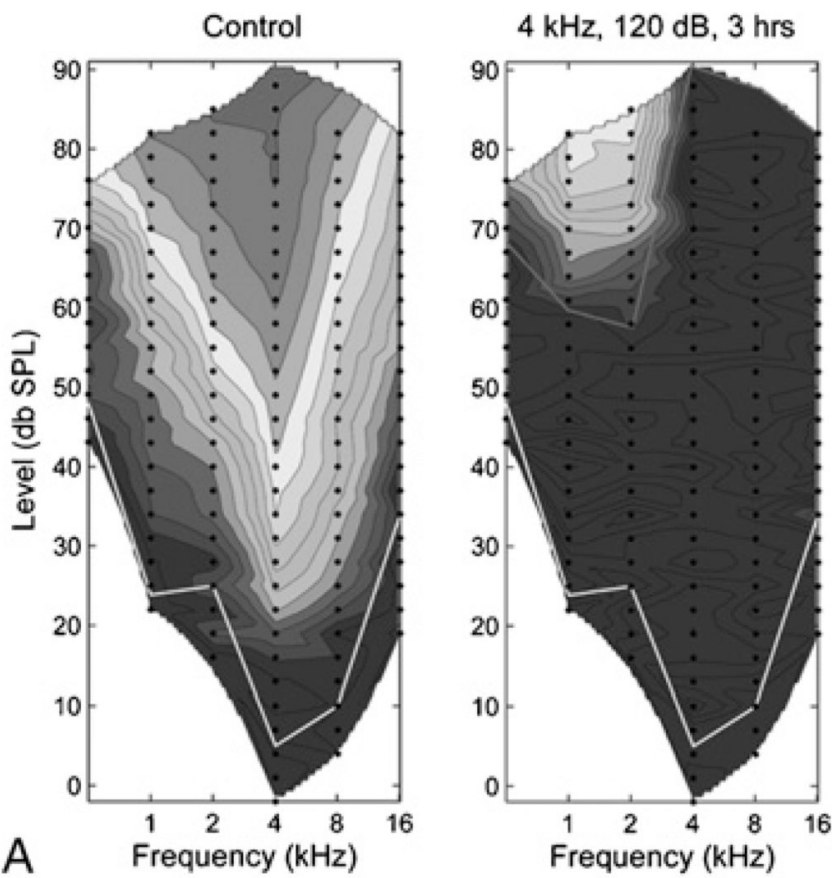

$2.5 \mathrm{kHz}, 120 \mathrm{~dB}, 3 \mathrm{hrs}$

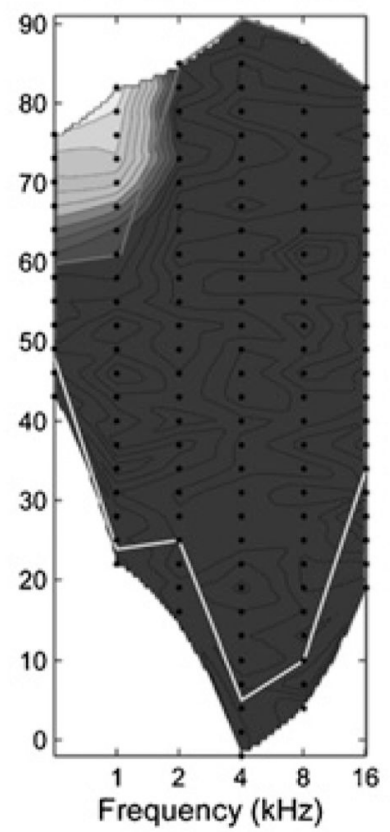

$2.0 \mathrm{kHz}, 116 \mathrm{~dB}, 3 \mathrm{hrs}$

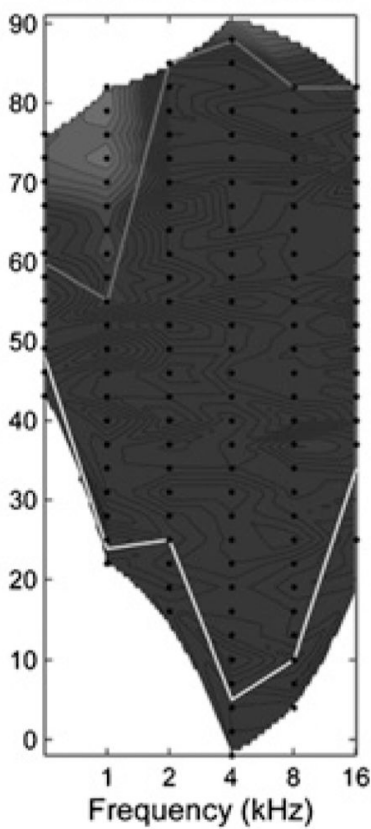

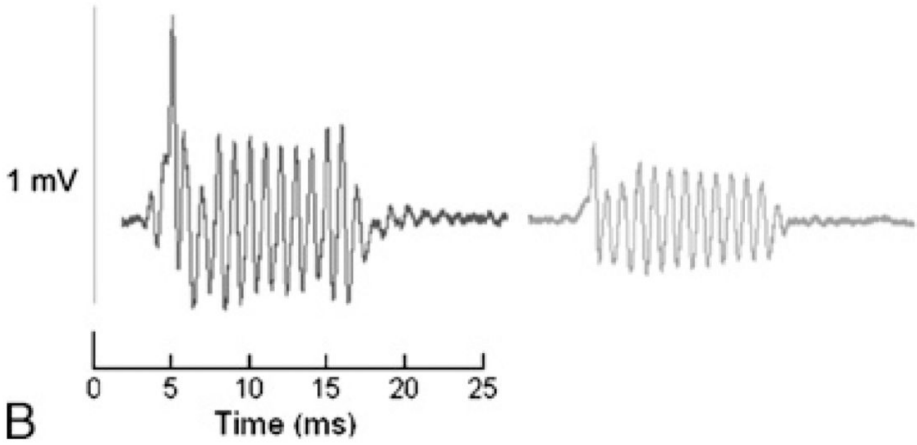
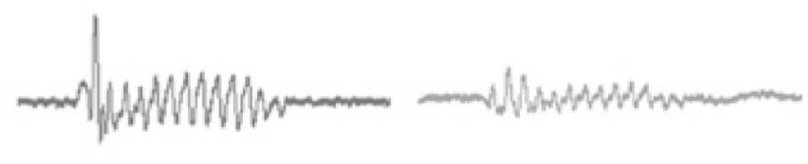

FIG. 5.

Near-field (round window) recordings. $\boldsymbol{A}$, Response area graphs representing CAP amplitudes for a control case and 3 noise exposure cases The color scale is from 25 (dark blue) to $60 \mathrm{~dB}$ (red), re $1 \mu \mathrm{V}$, with 15 equally spaced contours. Black dots are the stimuli presented, and lines are interpolated isoresponse contours. The white line indicates the threshold for the control ear, and the red lines are the threshold for each case). $\boldsymbol{B}$, Raw waveforms including the $\mathrm{CM}$ and CAP responses to a frequency stimulus at $1,000 \mathrm{~Hz}$ and $81 \mathrm{~dB}$ SPL, averaged over 100 stimuli. Importantly, even in the setting of profound hearing loss to high frequencies, near-field responses to preserved frequencies remain robust. 

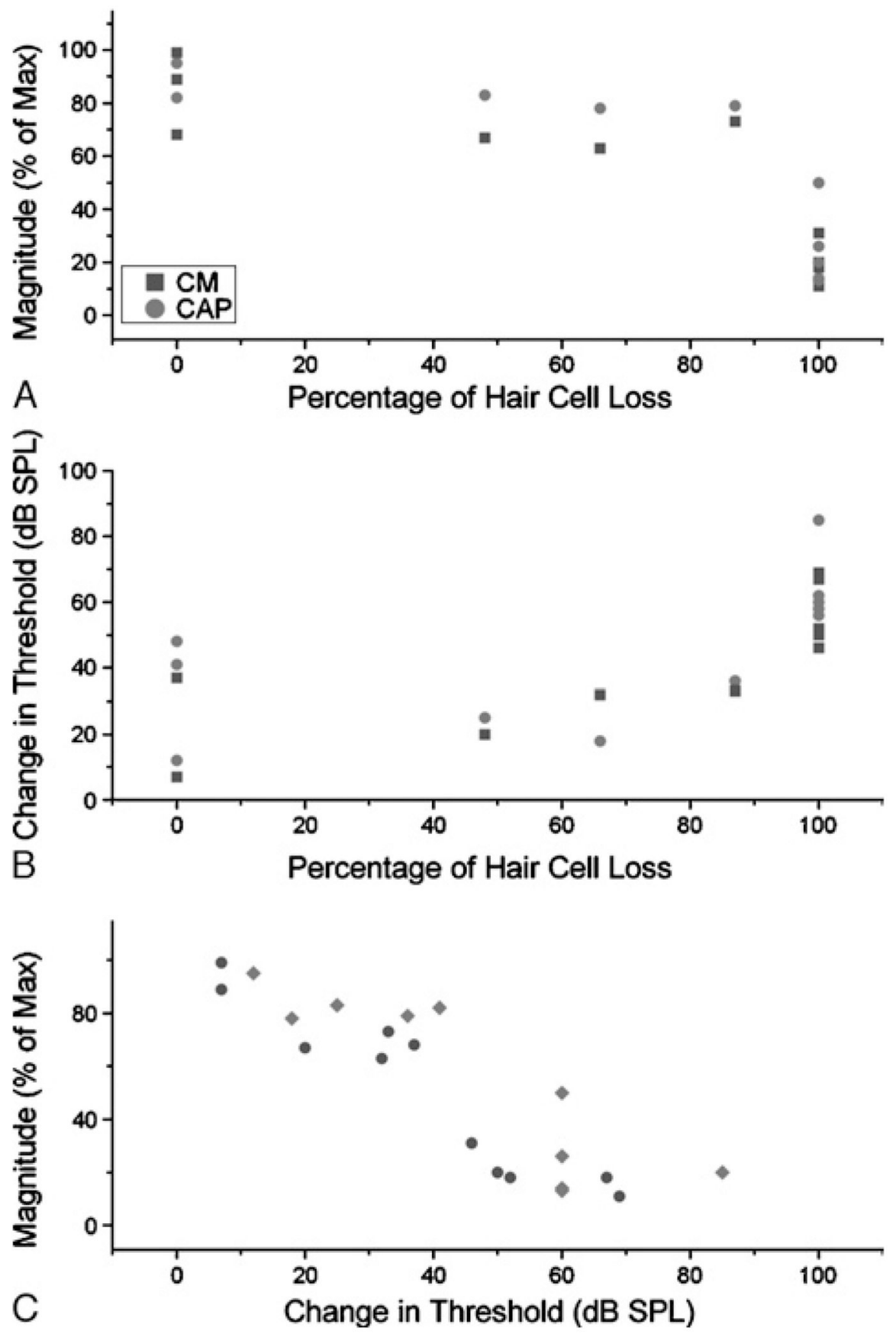

FIG. 6.

Correlations between anatomy and physiology. A, Change in magnitude of the CM and CAP as a function of hair cell loss. The hair cell loss was the percentage loss in hair cell counts from the most affected 250- $\mu \mathrm{m}$ portion of the cochlea, compared with the comparable location in the control ears. The change in magnitude was measured at the frequency closest to the cutoff frequency in each case and compared with a normal-hearing animal at the maximum level tested for that frequency. $\boldsymbol{B}$, Change in threshold of the CM and CAP. Threshold also was measured at the frequency closest to the cutoff frequency in each case and compared with a normal-hearing animal. $\boldsymbol{C}$, Relationship between the change in the magnitudes and thresholds. 


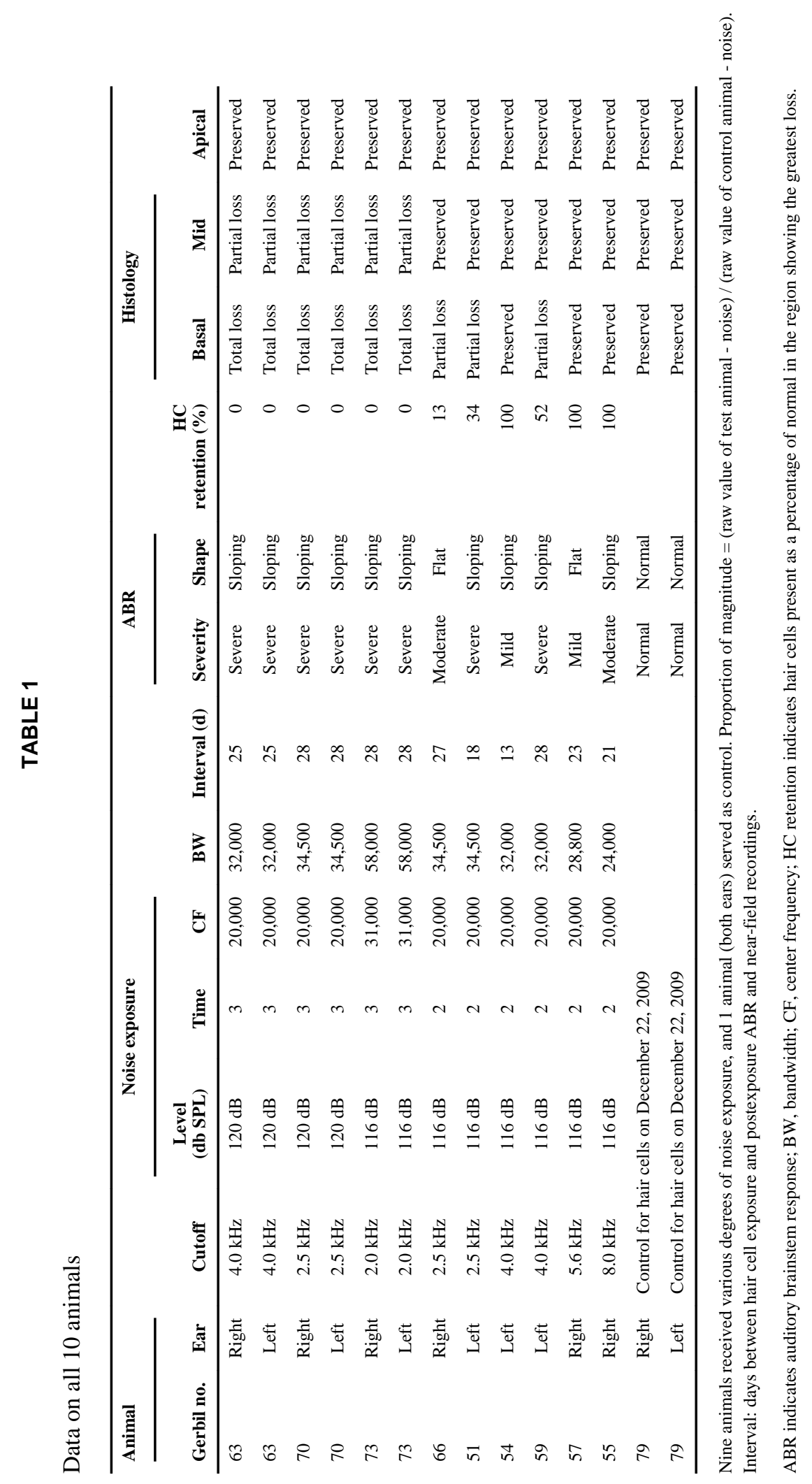

Otol Neurotol. Author manuscript; available in PMC 2012 June 1. 